\title{
The Cosmic Equation of State
}

\author{
F. Melia ${ }^{1}$
}

\begin{abstract}
The cosmic spacetime is often described in terms of the Friedmann-Robertson-Walker (FRW) metric, though the adoption of this elegant and convenient solution to Einstein's equations does not tell us much about the equation of state, $p=w \rho$, in terms of the total energy density $\rho$ and pressure $p$ of the cosmic fluid. $\Lambda \mathrm{CDM}$ and the $R_{\mathrm{h}}=c t$ Universe are both FRW cosmologies that partition $\rho$ into (at least) three components, matter $\rho_{\mathrm{m}}$, radiation $\rho_{\mathrm{r}}$, and a poorly understood dark energy $\rho_{\text {de }}$, though the latter goes one step further by also invoking the constraint $w=-1 / 3$. This condition is apparently required by the simultaneous application of the Cosmological principle and Weyl's postulate. Model selection tools in one-on-one comparisons between these two cosmologies favor $R_{\mathrm{h}}=c t$, indicating that its likelihood of being correct is $\sim 90 \%$ versus only $\sim 10 \%$ for $\Lambda \mathrm{CDM}$. Nonetheless, the predictions of $\Lambda \mathrm{CDM}$ often come quite close to those of $R_{\mathrm{h}}=c t$, suggesting that its parameters are optimized to mimic the $w=-1 / 3$ equation-of-state. In this paper, we explore this hypothesis quantitatively and demonstrate that the equation of state in $R_{\mathrm{h}}=c t$ helps us to understand why the optimized fraction $\Omega_{\mathrm{m}} \equiv \rho_{m} / \rho$ in $\Lambda$ CDM must be $\sim 0.27$, an otherwise seemingly random variable. We show that when one forces $\Lambda \mathrm{CDM}$ to satisfy the equation of state $w=\left(\rho_{\mathrm{r}} / 3-\rho_{\mathrm{de}}\right) / \rho$, the value of the Hubble radius today, $c / H_{0}$, can equal its measured value $c t_{0}$ only with $\Omega_{\mathrm{m}} \sim 0.27$ when the equation-of-state for dark energy is $w_{\mathrm{de}}=-1$. (We also show, however, that the inferred values of $\Omega_{\mathrm{m}}$ and $w_{\text {de }}$ change in a correlated fashion if dark energy is not a cosmological constant, so that $w_{\mathrm{de}} \neq-1$.) This peculiar value of $\Omega_{\mathrm{m}}$ therefore
\end{abstract}

\section{F. Melia}

Department of Physics, the Applied Math Program, and Department of Astronomy, The University of Arizona, Tucson, AZ 85721

E-mail: fmelia@email.arizona.edu

${ }^{1}$ John Woodruff Simpson Fellow. appears to be a direct consequence of trying to fit the data with the equation of state $w=\left(\rho_{\mathrm{r}} / 3-\rho_{\text {de }}\right) / \rho$ in a Universe whose principal constraint is instead $R_{\mathrm{h}}=c t$ or, equivalently, $w=-1 / 3$.

Keywords cosmic microwave background; cosmological parameters; cosmology: observations; cosmology: redshift; cosmology: theory; cosmology: dark matter; gravitation

\section{Introduction}

The Cosmological principle and Weyl's postulate appear to be essential ingredients in any physically realistic cosmological theory. Together, they posit that the Universe is homogeneous and isotropic (at least on large, i.e., > $100 \mathrm{Mpc}$, spatial scales), and that this high degree of symmetry is maintained from one time slice to the next. The appropriate spacetime to use is conveniently and elegantly written in terms of the Friedmann-Robertson-Walker (FRW) metric though this, in and of itself, does not tell us much about the cosmic equation of state, relating the total energy density $\rho$ to its total pressure $p$.

In principle, if we knew these quantities precisely, we could then solve the dynamical equations governing the Universal expansion and understand its large-scale structure and how it evolved to its current state. One could then also unambiguously interpret many of the observations, including the redshift-dependent luminosity distance to Type Ia SNe and the spectrum of fluctuations in the cosmic microwave background (CMB). Unfortunately, we must rely on measurements and intuition to pick $\rho$ and $p$. The best we can do today is to assume that $\rho$ must contain matter $\rho_{\mathrm{m}}$ and radiation $\rho_{\mathrm{r}}$, which we see directly, and an as yet poorly understand 'dark' energy $\rho_{\text {de }}$, whose presence is required by a 
broad range of data including, and especially, the aforementioned Type Ia SNe (Riess et al. 1998; Perlmutter et al. 1999). But instead of refining the cosmic equation of state, $p=w \rho$, the ever-improving measurements of the redshift-distance and redshift-age relations seem to be creating more tension between theory and observations, rather than providing us with a better indication of the dark-energy component, $p_{\mathrm{de}}=w_{\mathrm{de}} \rho_{\mathrm{de}}$. For the other two constituents, one simply uses the prescription $p_{\mathrm{r}}=\rho_{\mathrm{r}} / 3$ and $p_{\mathrm{m}} \approx 0$, consistent with a fully relativistic fluid (radiation) on the one hand, and a non-relativistic fluid (matter) on the other.

One of the most basic FRW models, $\Lambda$ CDM, assumes that dark energy is a cosmological constant $\Lambda$ with $w_{\mathrm{de}} \equiv w_{\Lambda}=-1$, and therefore $w=\left(\rho_{\mathrm{r}} / 3-\rho_{\Lambda}\right) / \rho$. This model does quite well explaining many of the observations, but growing empirical evidence suggests that it is inadequate to explain all of the nuances seen in cosmic evolution and the growth of structure. For example, $\Lambda \mathrm{CDM}$ cannot account for the general uniformity of the CMB across the sky without invoking an early period of inflated expansion (Guth 1981; Linde 1982), yet the latest observations with Planck (Ade et al. 2013) suggest that the inflationary model may be in trouble at a fundamental level (Ijjas et al. 2013, 2014; Guth et al. 2013). Insofar as the CMB fluctuations measured with both WMAP (Bennett et al. 2003) and Planck are concerned, there appears to be unresolvable tension between the predicted and measured angular correlation function (Copi et al. 2009, 2013; Melia 2014a; Bennett et al. 2013). And there is also an emerging conflict between the observed matter distribution function, which is apparently scale-free, and that expected in $\Lambda \mathrm{CDM}$, which has a different form on different spatial scales. The fine tuning required to resolve this difference led Watson et al. (Watson et al. 2011) to characterize the matter distribution function as a 'cosmic coincidence.' Such difficulties are compounded by $\Lambda$ CDM's predicted redshift-age relation, which does not appear to be consistent with the growth of quasars at high redshift (Melia 2013a), nor the very early appearance of galaxies at $z \gtrsim 10$ (Melia 2014b).

It is therefore important to refine the basic $\Lambda \mathrm{CDM}$ model, or perhaps to eventually replace it if necessary, to improve the comparison between theory and observations. Over the past several years, we have been developing another FRW cosmology, known as the $R_{\mathrm{h}}=c t$ Universe, that has much in common with $\Lambda$ CDM, but includes an additional ingredient motivated by several theoretical and observational arguments (Melia 2007; Melia \& Abdelqader 2009; Melia \& Shevchuk 2012). Like $\Lambda$ CDM, it also adopts the equation of state $p=w \rho$, with $p=p_{\mathrm{m}}+p_{\mathrm{r}}+p_{\mathrm{de}}$ and $\rho=\rho_{\mathrm{m}}+\rho_{\mathrm{r}}+\rho_{\mathrm{de}}$, but is subject to the additional constraint that $w=\left(\rho_{\mathrm{r}} / 3+w_{\mathrm{de}} \rho_{\mathrm{de}}\right) / \rho=-1 / 3$ at all times. One might come away with the impression that these two prescriptions for the equation of state cannot be consistent. But in fact if we ignore the constraint $w=-1 / 3$ and instead proceed to optimize the parameters in $\Lambda \mathrm{CDM}$ by fitting the data, the resultant value of $w$ averaged over a Hubble time is actually $-1 / 3$ within the measurement errors (Melia 2007; Melia \& Shevchuk 2012). In other words, though $w=\left(\rho_{\mathrm{r}} / 3-\rho_{\Lambda}\right) / \rho$ in $\Lambda \mathrm{CDM}$ cannot be equal to $-1 / 3$ from one moment to the next, its value averaged over the age of the Universe is equal to what it would have been in $R_{\mathrm{h}}=c t$ anyway.

This result does not necessarily prove that $\Lambda \mathrm{CDM}$ is an incomplete version of $R_{\mathrm{h}}=c t$, but it does seem to suggest that the inclusion of the additional constraint $w=-1 / 3$ might render its predictions closer to the data. By now, comparative analyses of $\Lambda \mathrm{CDM}$ and $R_{\mathrm{h}}=c t$ have been carried out for a broad range of observations, from the CMB (Melia 2014a), high- $z$ quasars (Melia 2013a, 2014b) and the ages of high- $z$ objects (Melia 2014b; Yu \& Wang 2014) in the early Universe, to gamma-ray bursts (Wei et al. 2013a) and cosmic chronometers (Melia \& Maier 2013) at intermediate redshifts and, most recently, to the relatively nearby Type Ia SNe (Wei et al. 2013b). In every case, model selection tools indicate that the likelihood of $R_{\mathrm{h}}=c t$ being correct is typically $\sim 90 \%$ compared with only $\sim 10 \%$ for $\Lambda \mathrm{CDM}$. And perhaps the most important distinguishing feature between these two cosmologies is that, whereas $\Lambda \mathrm{CDM}$ cannot survive without inflation, the $R_{\mathrm{h}}=c t$ Universe does not need it in order to avoid the well-known horizon problem (Melia 2014c). Thus, an eventual abandonment of inflation should it fail to work self-consistently would completely tip the scale in favor of $R_{\mathrm{h}}=c t$.

The purpose of this paper is to further develop the $R_{\mathrm{h}}=c t$ Universe by addressing a rather obvious question that comes to mind. Since $\Lambda \mathrm{CDM}$ lacks the ingredient $w=-1 / 3$ that would turn it into $R_{\mathrm{h}}=c t$, why does it in fact do quite well in accounting for many of the data? And are there any other obvious observational consequences of the prescription $w=$ $\left(\rho_{\mathrm{r}} / 3-\rho_{\Lambda}\right) / \rho$ for its equation of state? Here, we demonstrate that the inclusion of the condition $w=-1 / 3$ in $\Lambda \mathrm{CDM}$ actually helps to explain why the fraction $\Omega_{\mathrm{m}} \equiv \rho_{m}\left(t_{0}\right) / \rho\left(t_{0}\right)$ of its energy density in the form of (visible and dark) matter today must be $\approx 0.27$ in order for it to adequately fit the data. In other words, we will show that the inferred value of $\Omega_{\mathrm{m}}$ in $\Lambda \mathrm{CDM}$ is not random at all, but is instead uniquely required when one attempts to account for the observations using the equation of state $w=\left(\rho_{\mathrm{r}} / 3-\rho_{\Lambda}\right) / \rho$ 
in a Universe that is in reality evolving according to the constraint $w=\left(\rho_{\mathrm{r}} / 3+w_{\mathrm{de}} \rho_{\mathrm{de}}\right) / \rho=-1 / 3$. We will demonstrate this interesting and important connection between $\Lambda$ CDM and $R_{\mathrm{h}}=c t$ in $\S \S 2$ and 3 , and discuss the results in $\S 4$.

\section{The Cosmic Spacetime}

The basic $\Lambda$ CDM model avoids having to deal with uncertainties in the particle physics by relying on transitions, starting with an early radiation-dominated phase, followed by a Universe dominated by matter after recombination, and then transitioning into a period dominated by dark energy. But in order to make testable predictions, we have to assume values for $\Omega_{\mathrm{m}}$, $\Omega_{\mathrm{r}}$ and $\Omega_{\Lambda}$, and then integrate backwards to the big bang by solving the dynamics equations using the equation of state $w=\left(\rho_{\mathrm{r}} / 3-\rho_{\Lambda}\right) / \rho$. If the Universe is truly subject to the constraint $w=-1 / 3$ at all times, however, how does this affect the observable signatures and inferred parameters of the standard model? This is the question we will now attempt to answer.

The Friedmann-Robertson-Walker metric may be written

$$
\begin{array}{r}
d s^{2}=c^{2} d t^{2}-a^{2}(t)\left[d r^{2}\left(1-k r^{2}\right)^{-1}+\right. \\
\left.r^{2}\left(d \theta^{2}+\sin ^{2} \theta d \phi^{2}\right)\right],
\end{array}
$$

in terms of the cosmic time $t$ in the comoving frame, and the corresponding radial $(r)$ and angular $(\theta$ and $\phi)$ coordinates. The expansion factor $a(t)$ is a function of $t$ only, whereas the spatial coordinates $(r, \theta, \phi)$ in this frame remain "fixed" for all particles in the cosmos. The constant $k$ is +1 for a closed universe, 0 for a flat, open universe, or -1 for an open universe.

The source of spacetime curvature in a Universe that satisfies the Cosmological Principle is a perfect fluid Weinberg (1972) which, together with the metric coefficients appearing in Equation (1), allows us to simplify Einstein's equations and derive the key dynamical expressions governing the smoothed-out expansion at large scales. These include, respectively, the Friedmann and energy-conservation equations,

$H^{2} \equiv\left(\frac{\dot{a}}{a}\right)^{2}=\frac{8 \pi G}{3 c^{2}} \rho-\frac{k c^{2}}{a^{2}}$,

and

$\dot{\rho}=-3 H(\rho+p)$,

both written in terms of the total energy density $\rho$ and total pressure $p . H$ is the time-dependent Hubble 'constant' and an overdot denotes a derivative with respect to time $t$.
In the $R_{\mathrm{h}}=c t$ Universe, the 'active mass' is zero, meaning that $\rho+3 p=0$ (Melia 2014d). Therefore, from the definition of the gravitational radius $R_{\mathrm{h}}=2 G M / c^{2}$, in terms of the Misner-Sharp mass $M=(4 \pi / 3) R_{\mathrm{h}}^{3}\left(\rho / c^{2}\right)$ (Misner \& Sharp 1964), it is easy to show that $\dot{R}_{\mathrm{h}}=(3 / 2)(1+w) c$, where $w=p / \rho$ (Melia \& Shevchuk 2012), which yields $R_{\mathrm{h}}=c t$ (the eponymous constraint of this model). And since the gravitational radius $R_{\mathrm{h}}$ is a proper distance in this spacetime, one must also have $H=1 / t$ (see, e.g., Melia \& Shevchuk 2012) which, together with Equation (2), then shows that $k=0$.

Interestingly the CMB strongly constrains the total energy density to be near its critical value, $\rho_{c} \equiv$ $3 c^{2} H_{0}^{2} / 8 \pi G$, where $H_{0} \equiv H\left(t_{0}\right)$ (Bennett et al. 2003; Spergel et al. 2003), so the observations appear to be consistent with zero spatial curvature. Though this empirical result emerges from the optimization of model parameters in $\Lambda \mathrm{CDM}$, the fact that the standard model is often a good approximation to $R_{\mathrm{h}}=c t$ lends some observational support for this theoretical prediction of the $R_{\mathrm{h}}=c t$ cosmology. For these reasons, it will be sensible for us to assume a perfectly flat universe, and we will here always assume that $k=0$. This also means that $\Omega \equiv \Omega_{\mathrm{r}}+\Omega_{\mathrm{m}}+\Omega_{\mathrm{de}}=1$. Our analysis in this paper will be based entirely on this premise. It is therefore straightforward to integrate Equation (2), yielding

$c t_{0}=R_{\mathrm{h}}\left(t_{0}\right) \int_{0}^{1} \frac{u d u}{\sqrt{\Omega_{\mathrm{r}}+\Omega_{\mathrm{m}} u+\Omega_{\mathrm{de}} u^{1-3 w_{\mathrm{de}}}}}$.

To obtain this expression, we have allowed for the possibility that dark energy is not a cosmological constant (i.e., that $w_{\text {de }}$ may be different from -1 , in which case we would refer to this model as wCDM, rather than $\Lambda \mathrm{CDM}$ ), and we have used the derived value of the gravitational horizon to write $R_{\mathrm{h}}=c / H$ (Melia 2007; Melia \& Shevchuk 2012). This expression also assumes that $a \rightarrow 0$ at $t=0$.

Equation (4) must be satisfied by every flat FRW cosmology, though the explicit dependence of the integrand on $\Omega_{\mathrm{m}}, \Omega_{\mathrm{r}}$, and $\Omega_{\mathrm{de}}$ shown here applies specifically to $\Lambda \mathrm{CDM}$ (or $\mathrm{wCDM}$ if $w_{\mathrm{de}} \neq-1$ ). However, if in fact the Cosmological Principle and Weyl's postulate require the equation of state $w=-1 / 3$, then $R_{\mathrm{h}}\left(t_{0}\right)=c t_{0}$ (Melia 2007; Melia \& Shevchuk 2012), so $\Lambda \mathrm{CDM}$ (or wCDM) would have no choice but to satisfy the condition

$\mathcal{I} \equiv \int_{0}^{1} \frac{u d u}{\sqrt{\Omega_{\mathrm{r}}+\Omega_{\mathrm{m}} u+\Omega_{\mathrm{de}} u^{1-3 w_{\mathrm{de}}}}}=1$.

Let us now see what the consequences of this constraint are for $\Lambda$ CDM. Figure 1 shows the calculated value of 
this integral $\mathcal{I}$ as a function of $\Omega_{\mathrm{m}}$, for various darkenergy equations of state, $w_{\text {de }}$. The radiation energy density is evaluated on the basis of the CMB's current temperature, $T=2.7 \mathrm{~K}$. Not surprisingly, $\mathcal{I}$ can have a broad range of values, but for any given $w_{\text {de }}$, there is only one unique determination of $\Omega_{\mathrm{m}}$ that satisfies the condition $\mathcal{I}=1$. And for the special case of a cosmological constant $\left(w_{\mathrm{de}}=-1\right)$, that value is 0.27 .

\section{Observational Constraints}

Over the past decade, both $\Omega_{\mathrm{m}}$ and $w_{\mathrm{de}}$ have been measured with relatively high precision, combining constraints from a variety of observational data sets. For example, Melchiorri et al. (2003) combined data from six CMB experiments (Spergel et al. 2003), from the power spectrum of large-scale structure in the $2 \mathrm{dF} 100 \mathrm{k}$ galaxy redshift survey (Tegmark et al. 2002), from luminosity measurements of Type Ia SNe (Riess et al. 1998; Perlmutter et al. 1999), and from the Hubble space telescope measurements of the Hubble parameter $H_{0}$. More recent analyses have refined the quantitative results from this extensive survey, though not altering the basic conclusions. It is therefore rather straightforward for us to compare our theoretical predictions directly with the observations. As we shall see shortly, the story emerging from this exercise is quite revealing.

None of the individual observations results in fits that are so precise as to produce unique values for the parameters $\left(w_{\mathrm{de}}, \Omega_{\mathrm{m}}\right)$. The reason for this is that, other than the Sachs-Wolfe effect (Sachs \& Wolfe 1967), which is responsible for the largest angular fluctuations in the $\mathrm{CMB}$, none of the other mechanisms producing structure of one kind or another depends sensitively on the expansion history of the Universe. As such, some degeneracy exists among the possible choices of cosmological parameters pertaining to the CMB (Kosowsky et al. 2002).

At lower redshifts, the cosmological measurements are heavily influenced by the observation of Type Ia SNe. But here also, both the luminosities and angular distances (the fundamental observables) depend on $w_{\text {de }}$ through multiple integrals, and are therefore not particularly sensitive to variations in $w_{\text {de }}$ with redshift (Maor et al. 2001).

Nonetheless, all of the constraints derived from the various data sets do produce a well-defined region in $w_{\text {de }}-\Omega_{\mathrm{m}}$ phase space where the most likely values of these parameters are expected to be found. The confidence regions shown in figure 2 are adapted from a corresponding figure in Melchiorri et al. (2003). These show the $68 \%, 95 \%$, and $99 \%$ confidence regions corresponding to the Type Ia SNe observations (adapted

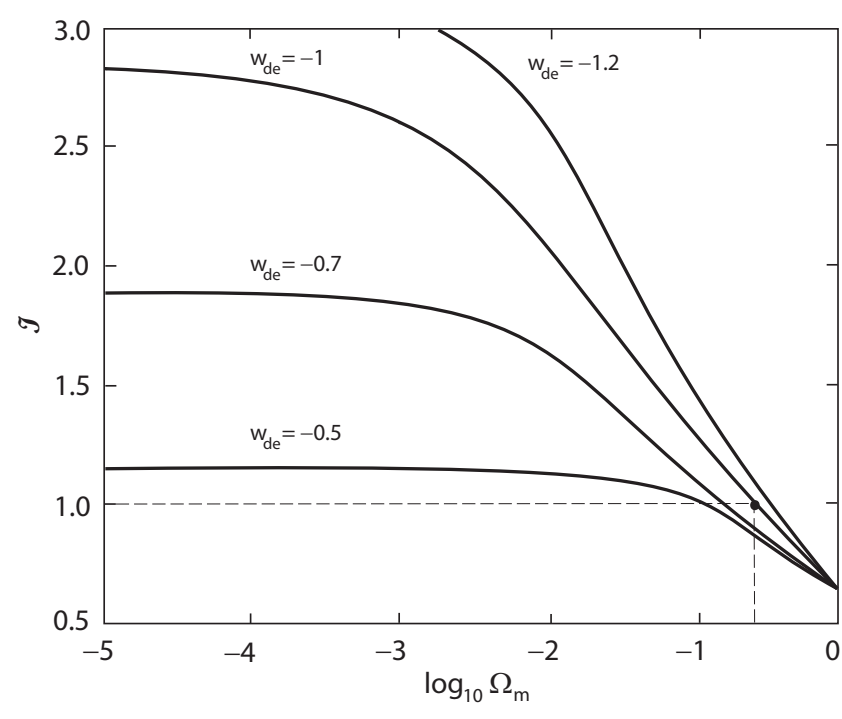

Fig. 1 The ratio $\mathcal{I} \equiv c t_{0} / R_{\mathrm{h}}\left(t_{0}\right)$, calculated as a function of $\Omega_{\mathrm{m}}$, according to $\Lambda \mathrm{CDM}$ (or ${ }_{\mathrm{wCDM}}$ when $w_{\mathrm{de}} \neq-1$ ). The label $w_{\text {de }}$ indicates the corresponding equation of state for dark energy, i.e., $p_{\mathrm{de}}=w_{\mathrm{de}} \rho_{\mathrm{de}}$, in terms of its pressure $p_{\text {de }}$ and density $\rho_{\text {de }}$. When $R_{\mathrm{h}}\left(t_{0}\right)=c t_{0}$ and $w_{\text {de }}=-1$, $\Omega_{\mathrm{m}}$ must have the unique value 0.27 (indicated by the black dot).

from Suzuki et al. 2012, shown as gray swaths), and the corresponding regions inferred from the analysis of $\mathrm{CMB}$, HST, and $2 \mathrm{dF}$ data (indicated by the lightercolored island regions to the upper left of this diagram). Insofar as the values of $w_{\mathrm{de}}$ and $\Omega_{\mathrm{m}}$ are concerned, the supernova data are not as constraining as the other sets, but there is clearly a satisfying consistency among all of the observations.

Also shown in figure 2 is one of the more interesting results of this paper, indicated here as a thick black curve to the left of this diagram. This feature shows the loci of $\left(w_{\mathrm{de}}, \Omega_{\mathrm{m}}\right)$ points permitted by the requirement that the integral $\mathcal{I}$ be equal to 1 (see also figure 1 ). That is, while the constraints shown in figure 2 are based on the interpretation of the data using $\Lambda \mathrm{CDM}$, this theoretical curve goes one step further, by illustrating what values of $w_{\text {de }}$ and $\Omega_{\mathrm{m}}$ are actually permitted theoretically when we impose the additional constraint $R_{\mathrm{h}}\left(t_{0}\right)=c t_{0}$ (or, equivalently, the equation of state $w=-1 / 3)$. Notice, for example, where the latest measurement of $\Omega_{\mathrm{m}}$ and $w_{\mathrm{de}}$ with Planck fall on this diagram (the star in figure 2). Whereas $\Omega_{\mathrm{m}}=0.27$ is linked to a dark-energy equation of state $w_{\mathrm{de}}=-1$, the Planck measurement of $\Omega_{\mathrm{m}} \approx 0.3$ is associated with $w_{\text {de }}=1.13_{-0.10}^{+0.13}$ (Ade et al. 2013).

On its own, $\Lambda \mathrm{CDM}$ has no explanation for why the most preferred region of allowed values is limited to $-1.38<w_{\mathrm{de}}<-0.82$ and $0.22<\Omega_{\mathrm{m}}<0.35$, and why 
this oblong region is slanted in such a way as to couple the higher values of $w_{\mathrm{de}}$ to the smaller values of $\Omega_{\mathrm{m}}$. But in the context of $R_{\mathrm{h}}=c t$, this is precisely the region permitted by the requirement that $\mathcal{I}$ be equal to 1 , as evidenced by the fact that our theoretical curve passes directly through the middle of the observationally permitted region and, even more impressively, precisely tracks the orientation of this region. The point of this is that while the data are not sufficiently precise to tell us the exact value of $\Omega_{\mathrm{m}}$, the range of allowed values of $w_{\mathrm{de}}$ trends with $\Omega_{\mathrm{m}}$ in such a way as to always preserve the condition $\mathcal{I}=1$.

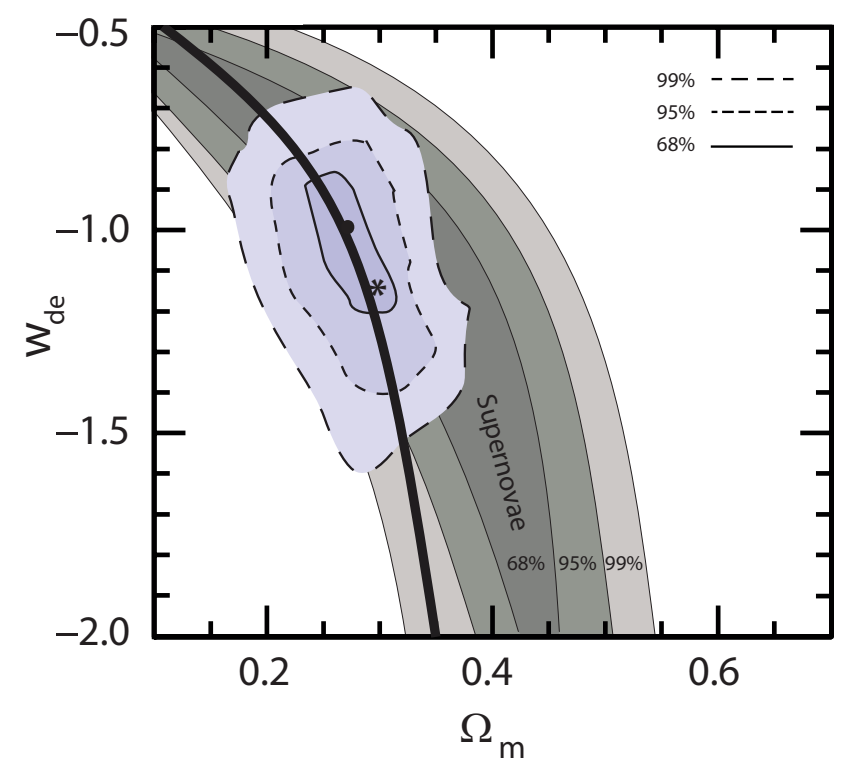

Fig. 2 The solid black curve indicates the value $w_{\text {de }}$ must have in $\Lambda \mathrm{CDM}$ (or ${ }_{\mathrm{wCDM}}$ if $w_{\mathrm{de}} \neq-1$ ) as a function of $\Omega_{\mathrm{m}}$, when the condition $R_{\mathrm{h}}\left(t_{0}\right)=c t_{0}$ is imposed. This curve is shown against the constraints (adapted from figure 4 in Melchiorri et al. 2003) on the dark-energy equationof-state, assuming a flat universe. The Type Ia SN limits have been updated from the more recent results in Suzuki et al. (2012). These limits and confidence levels include results from CMB anisotropies, measurements of the Hubble constant, and large-scale structure. The empirically derived, concordance values of $w_{\text {de }}$ versus $\Omega_{\mathrm{m}}$ track those imposed on $\Lambda$ CDM by the $R_{\mathrm{h}}\left(t_{0}\right)=c t_{0}$ condition exceptionally well. Note, for example, the location (black dot) of the WMAP measurements (Bennett et al. 2013), versus (star) the latest measurements by Planck (Ade et al. 2013), which resulted in the values $\Omega_{\mathrm{m}} \approx 0.3$ and $w_{\mathrm{de}} \approx-1.13$. The value $\Omega_{\mathrm{m}}=0.27$ is realized only when $w_{\mathrm{de}}=-1$.

These results clearly argue against any suggestion that $\Omega_{\mathrm{m}} \sim 0.3$ (or, more specifically, $\Omega_{\mathrm{m}}=0.27$ when $w_{\mathrm{de}}=-1$ ) could be a coincidence in $\Lambda$ CDM. First, not only would it be highly improbable for $\Omega_{\mathrm{m}}$ to have the value required to guarantee $R_{\mathrm{h}}\left(t_{0}\right)=c t_{0}$ which, by the way, could only happen once in the entire history of the
Universe, and it would have to be happening right now, when we just happen to be looking. But in addition, the region of $w_{\mathrm{de}}-\Omega_{\mathrm{m}}$ phase space permitted by the data shows a clear trend exactly matching the behavior one would expect if $\mathcal{I}$ must always be equal to 1 . In other words, even if $\Omega_{\mathrm{m}} \sim 0.3$ were somehow a coincidence, there is no reason why the allowed region of $w_{\mathrm{de}}-\Omega_{\mathrm{m}}$ phase space should be slanted from upper left to bottom right, instead of from upper right to bottom left.

It is therefore difficult to argue against the conclusion that $\Lambda \mathrm{CDM}$ is merely mimicking the expansion history we would have obtained with $R_{\mathrm{h}}=c t$ all along, and that the observed value of $\Omega_{\mathrm{m}}$ (which happens to be 0.27 if $w_{\mathrm{de}}=-1$ ) is required in order to make the assumed density $\rho=\rho_{r}+\rho_{m}+\rho_{\text {de }}$ comply with the equation of state $p=-\rho / 3$ found in the $R=c t$ Universe.

\section{Discussion and Conclusion}

The results we have just presented do not exist in isolation, of course. They add weight to the other oneon-one comparisons between $R_{\mathrm{h}}=c t$ and $\Lambda \mathrm{CDM}$ that uniformly show the superiority of the former over the latter in accounting for the data. But the analysis we have carried out in this paper is important specifically because it starts to probe the fundamental reasons why $\Lambda \mathrm{CDM}$ can sometimes function as an approximation to $R_{\mathrm{h}}=c t$, and why it does reasonably well accounting for some of the data, e.g., the Type Ia SNe. For example, even though the empirically motivated choice of density $\rho=\rho_{r}+\rho_{m}+\rho_{\text {de }}$ is not entirely consistent with the equation of state $p=-\rho / 3$, it can nonetheless lead to an expansion history that mimics $R_{\mathrm{h}}=c t$ over a Hubble time - but only so long as $\Omega_{\mathrm{m}} \sim 0.27$.

Recently, we studied in detail how the Type Ia SNe ought to be interpreted in the context of $\Lambda \mathrm{CDM}$ and $R_{\mathrm{h}}=c t$ (Melia 2012a; Wei et al. 2013b). The best-fit distance moduli calculated from these two theories are so close to each other all the way out to $z \sim 6$, that it is difficult to determine on the basis of a $\chi^{2}$ comparison alone which of these two cosmologies is favored. This is due in part to the strong dependence of the data reduction itself on the pre-assumed cosmology, since at least 4 'nuisance' parameters defining the SN luminosity must be optimized along with the free parameters of the model. The inferred SN luminosities and their 'measured' distance moduli are therefore strongly compliant to the pre-assumed model, greatly weakening this particular comparative test. Indeed, a similar analysis of the most up-do-date Gamma-ray Burst Hubble Diagram (HD) (Wei et al. 2013a) reinforces this point by 
demonstrating that when the data are re-calibrated correctly for each individual cosmology, the $R_{\mathrm{h}}=c t$ Universe fits the observed HD better than $\Lambda$ CDM does.

A quick inspection of figure 3 allows us to better appreciate why $\Lambda \mathrm{CDM}$ fits the Type Ia SNe and Gammaray Burst data as well as it does. This figure shows the ratio of luminosity distances $d_{L}^{\Lambda \mathrm{CDM}} / d_{L}^{R_{\mathrm{h}}=c t}$ as a function of redshift for different values of $\Omega_{\mathrm{m}}$, in a Universe with $w_{\mathrm{de}}=-1$. What emerges from this diagram is that the value of $\Omega_{\mathrm{m}}$ that comes closest to satisfying the condition $R_{\mathrm{h}}\left(t_{0}\right)=c t_{0}$ in Equation (4), also corresponds to the $\Lambda \mathrm{CDM}$ universe in which the luminosity distance $d_{L}^{\Lambda \mathrm{CDM}}$ most closely tracks its counterpart in $R_{\mathrm{h}}=c t$. One should not be surprised therefore, to see that the best fit $\Lambda \mathrm{CDM}$ cosmology fits the Type Ia SNe and Gamma-ray Burst data as well as it does.

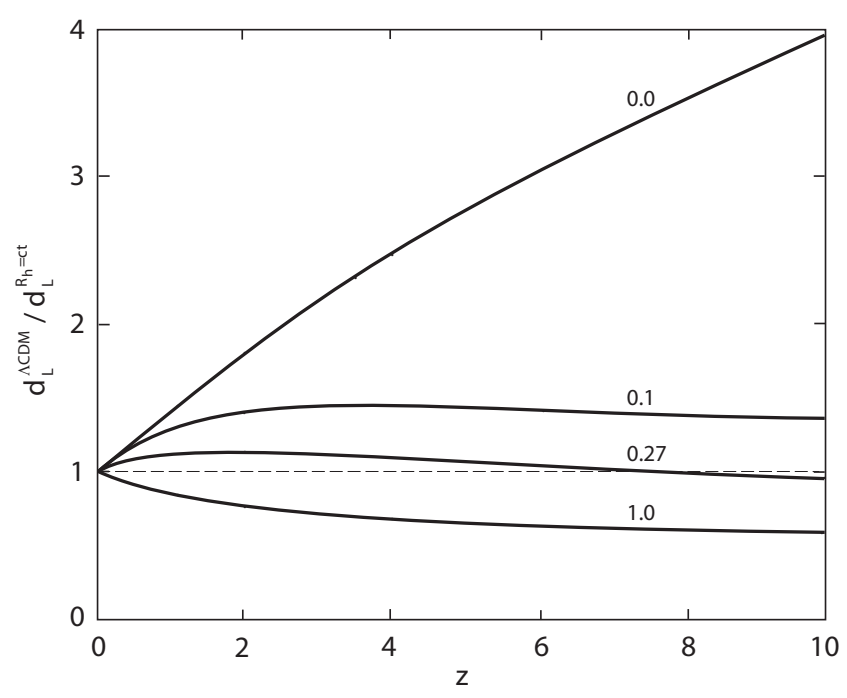

Fig. 3 Ratio of luminosity distance in $\Lambda$ CDM over that in $R_{\mathrm{h}}=c t$, as a function of redshift, for various values of $\Omega_{\mathrm{m}}$, assuming $w_{\mathrm{de}}=-1$. The $\Lambda$ CDM cosmology with $\Omega_{\mathrm{m}}=0.27$, which comes closest to satisfying the condition $R_{\mathrm{h}}\left(t_{0}\right)=c t_{0}$, also best approximates the condition $d_{L}^{\Lambda \mathrm{CDM}}=d_{L}^{R_{\mathrm{h}}=c t}$ over a large range in $z$.

Unfortunately, $\Lambda \mathrm{CDM}$ does not do as well accounting for the high- $z$ universe, having difficulty explaining why the CMB fluctuations show no correlation at angles greater than $\sim 60^{\circ}$, and failing to explain how $\sim 10^{9} M_{\odot}$ supermassive black holes could have formed so quickly after the big bang. The issue is that even though the differences between $\Lambda \mathrm{CDM}$ and $R_{\mathrm{h}}=c t$ may be smoothed out over a Hubble time through the careful choice of $\Omega_{\mathrm{m}} \sim 0.27$, the expansion history of the Universe at $z>6$ is so different between these two cosmologies that it is simply not possible to mimic the equation of state $p=-\rho / 3$ with $\rho=\rho_{r}+\rho_{m}+\rho_{\text {de }}$, given that $R_{\mathrm{h}}=$ ct predicts a constant expansion, while
$\Lambda \mathrm{CDM}$ predicts a very rapid deceleration. Additional inconsistencies between the predicted age-redshift relationship in $\Lambda \mathrm{CDM}$ and that observed for the oldest objects in the Universe have recently been pointed out by Yu \& Wang (2014).

And lest the reader come away with the sense that $\Lambda \mathrm{CDM}$ and $R_{\mathrm{h}}=c t$ overlap so much that one should not worry about their differences, we close this discussion by again pointing out the most profound consequence of their disparity. As shown in Melia (2014c), the horizon problem does not exist in $R_{\mathrm{h}}=$ ct. So whereas $\Lambda \mathrm{CDM}$ could not survive without inflation, the real universe may have done without it, and the cosmological data - particularly at high redshift - may be pointing in that direction.

Acknowledgements I am grateful to the anonymous referee for suggestions that have led to improvements in the manuscript. I am also grateful to Amherst College for its support through a John Woodruff Simpson Lectureship, and to Purple Mountain Observatory in Nanjing, China, for its hospitality while part of this work was being carried out. This work was partially supported by grant 2012T1J0011 from The Chinese Academy of Sciences Visiting Professorships for Senior International Scientists, and grant GDJ20120491013 from the Chinese State Administration of Foreign Experts Affairs. 


\section{References}

Ade, P.A.R. et al. (Planck Collaboration) 2013, A\&A, in press (arXiv:1303.5062)

Bennett, C. L. et al. 2003, ApJ, 583, 1

Bennett, C. L. et al. 2013, ApJS, 208, 20

Copi, C. J., Huterer, D., Schwarz, D. J. \& Starkman, G. D. 2009, MNRAS, 399, 295

Copi, C. J., Huterer, D., Schwarz, D. J. \& Starkman, G. D. 2013, MNRAS in press (arXiv:1310.3831)

Guth, A. H. 1981, PRD, 23, 347

Guth, A. H., Kaiser, D. I. \& Nomura, I. 2013, eprint arXiv: 1312.7619

Ijjas, A., Steinhardt, P. J. \& Loeb, A. 2013, PLB, 723, 261

Ijjas, A., Steinhardt, P. J. \& Loeb, A. 2013, eprint arXiv:1402.6980

Kosowsky, A., Milosavljevic, M. \& Jimenez, R. 2002, PRD, 66, 063007

Linde, A. 1982, PLB, 108, 389

Maor, I., Brustein, R. \& Steinhardt, P. J. 2001, PRL, 86, 6

Melchiorri, A., Mersini, L., Ödman, C. J. Trodden, M. 2003, PRD, 68, 043509

Melia, F. 2007, MNRAS, 382, 1917

Melia, F. 2012a, AJ, 144, id. 110

Melia, F. 2012b, JCAP, 09, 029

Melia, F. 2013, ApJ, 764, 72

Melia, F. 2013b, CQG, 30, 155007

Melia, F. 2014a, A\&A, 561, id A80

Melia, F. 2014b, AJ, 147, id 120

Melia, F. 2014c, A\&A, 553, id A76

Melia, F. 2014d, A\&A, 553, id A76

Melia, F. \& Abdelqader, M. 2009, IJMP-D, 18, 1889

Melia, F. \& Maier, R. S. 2013, MNRAS, 432, 2669

Melia, F. \& Shevchuk, A.S.H. 2012, MNRAS, 419, 2579

Misner, C. W. \& Sharp, D. H. 1964, Phys Rev, 136, 571

Perlmutter, S. et al. 1999, ApJ, 517, 565

Riess, A. G. et al. 1998, AJ, 116, 1009

Sachs, R. K. \& Wolfe, A. M. 1967, ApJ, 147, 73

Spergel, D. N. et al. 2003, ApJS, 148, 175

Suzuki, N., Rubin, D., Lidman, C., et al. 2012, ApJ, 746, 85

Tegmark, M., Hamilton, A.J.S. \& Xu, Y. 2002, MNRAS, 335,887

Watson, D. F., Berlind, A. A. \& Zentner, A. R. 2011, ApJ, 738 , id. 22

Wei, J.-J., Wu, X.-F. \& Melia, F. 2013a, ApJ, 772, 43

Wei, J.-J., Wu, X.-F., Melia, F. \& Maier, R. S. 2013b, AJ, in press

Weinberg, S. 1972, Gravitation and Cosmology: Principles and Applications of the General Theory of Relativity (Wiley, New York)

Yu, H. \& Wang, F. Y. 2014, EPJ-C, 74, id. 3090

This manuscript was prepared with the AAS LATEX macros v5.2. 